\title{
Optically thin hybrid cavity for terahertz photo-conductive detectors
}

R. J. Thompson, T. Siday, S. Glass, T. S. Luk, J. L. Reno, I. Brener, and O. Mitrofanov

Citation: Appl. Phys. Lett. 110, 041105 (2017); doi: 10.1063/1.4974482

View online: http://dx.doi.org/10.1063/1.4974482

View Table of Contents: http://aip.scitation.org/toc/apl/110/4

Published by the American Institute of Physics

\section{Articles you may be interested in}

The role of bandgap energy excess in surface emission of terahertz radiation from semiconductors Applied Physics Letters 110, 042101 (2017); 10.1063/1.4974479

Mid-IR colloidal quantum dot detectors enhanced by optical nano-antennas Applied Physics Letters 110, 041106 (2017); 10.1063/1.4975058

Detection of internal fields in double-metal terahertz resonators Applied Physics Letters 110, 061109 (2017); 10.1063/1.4975802

Terahertz plasmonic rectification in a spatially periodic graphene Applied Physics Letters 110, 061106 (2017); 10.1063/1.4975829

Monolayer graphene based organic optical terahertz modulator Applied Physics Letters 110, 023301 (2017); 10.1063/1.4973816

lon capturing/ion releasing films and nanoparticles in liquid crystal devices Applied Physics Letters 110, 041103 (2017); 10.1063/1.4974453

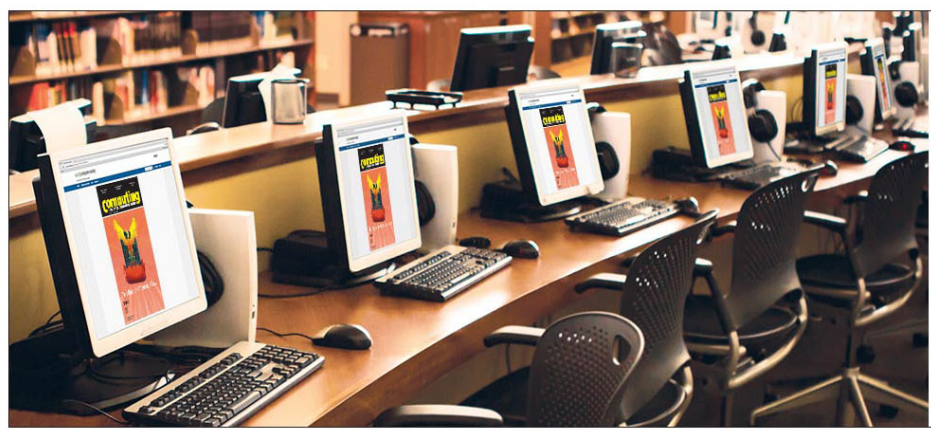

\section{CISE is already at} your fingertips...

\section{Computing}

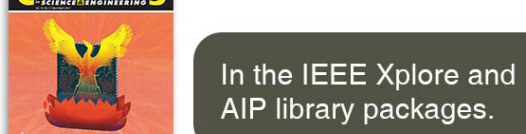

AIP library packages. 


\title{
Optically thin hybrid cavity for terahertz photo-conductive detectors
}

\author{
R. J. Thompson, ${ }^{1,2, a)}$ T. Siday, ${ }^{1}$ S. Glass, ${ }^{1}$ T. S. Luk, ${ }^{3,4}$ J. L. Reno, ${ }^{3,4}$ I. Brener, ${ }^{3,4}$ \\ and O. Mitrofanov ${ }^{1,3}$ \\ ${ }^{1}$ Electronic and Electrical Engineering, University College London, London WC1E 7JE, United Kingdom \\ ${ }^{2}$ London Center for Nanotechnology, University College London, London WC1H OAH, United Kingdom \\ ${ }^{3}$ Center for Integrated Technologies, Sandia National Laboratory, Albuquerque, New Mexico 87185, USA \\ ${ }^{4}$ Sandia National Laboratory, Albuquerque, New Mexico 87185, USA
}

(Received 25 August 2016; accepted 9 January 2017; published online 24 January 2017)

\begin{abstract}
The efficiency of photoconductive (PC) devices, including terahertz detectors, is constrained by the bulk optical constants of PC materials. Here, we show that optical absorption in a PC layer can be modified substantially within a hybrid cavity containing nanoantennas and a Distributed Bragg Reflector. We find that a hybrid cavity, consisting of a GaAs PC layer of just $50 \mathrm{~nm}$, can be used to absorb $>75 \%$ of incident photons by trapping the light within the cavity. We provide an intuitive model, which describes the dependence of the optimum operation wavelength on the cavity thickness. We also find that the nanoantenna size is a critical parameter, small variations of which lead to both wavelength shifting and reduced absorption in the cavity, suggesting that impedance matching is key for achieving efficient absorption in the optically thin hybrid cavities. Published by AIP Publishing. [http://dx.doi.org/10.1063/1.4974482]
\end{abstract}

Scientific investigations, ranging from research on quantum dots to astronomy, increasingly exploit terahertz $(\mathrm{THz})$ radiation. However, the efficiency of $\mathrm{THz}$ detectors and emitters remains a major research challenge. Nanoscale structures offer a wide range of approaches for mitigating this problem: quantum dots, ${ }^{1}$ nano-wires,${ }^{2,3}$ graphene, ${ }^{4,5}$ and topological insulators ${ }^{6}$ have been investigated as $\mathrm{THz}$ detectors, ${ }^{2,4}$ whereas plasmonic nanostructures have been exploited to enhance the sensitivity of photoconductive (PC) devices. ${ }^{7,8}$ The latter approach has opened doorways to improve one of the most widely used $\mathrm{THz}$ detectors, the ultrafast PC switch.

The PC switch operates as an opto-electronic transistor where a short pulse of photons turns on electrical conductivity within a channel. PC materials with sub-picosecond photocarrier lifetimes, such as low temperature grown GaAs, are used in the channel to enable detection of the $\mathrm{THz}$ field. However, the optical absorption length in GaAs is larger than the mean distance that the photo-carriers travel within the device. ${ }^{9,10}$ This limits the photocurrent and temporal resolution of the switch. ${ }^{11}$ Nanostructured plasmonic electrodes were shown to improve the collection of photocarriers; ${ }^{7,12-16}$ however, they are not ideal for $\mathrm{THz}$ detectors as the narrow spacing between them can lead to an increase in dark current and subsequently detector noise. To address this issue, the concept of light trapping, ${ }^{17}$ which has been extensively investigated for thin film photovoltaics ${ }^{18-20}$ and detectors, ${ }^{21}$ was recently implemented in THz detectors, ${ }^{8}$ where an optically thin hybrid cavity was used to improve detection sensitivity. Light trapping within the cavity relaxes the requirement that the $\mathrm{PC}$ region must be thicker than the absorption length in GaAs, and enables optically thin PC THz detectors. ${ }^{22}$ In this paper, we provide insight into effects of the hybrid cavity design on optical absorption. We find that a hybrid cavity with a PC layer of only $50 \mathrm{~nm}, 1 / 15$ of the absorption length in bulk GaAs, provides overall optical

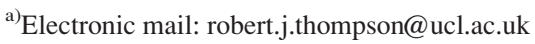

absorption of over $75 \%$. Our detailed investigation indicates that matching the impedance of the nanoantenna array to the free space impedance is a key condition for the enhanced absorption in the hybrid cavity. Furthermore, numerical simulations of the hybrid cavity allow us to evaluate relative contributions of the PC layer and the nanoantennas in the overall absorption.

The cavity investigated here contains a nanoantenna array placed above an optically thin PC layer and a Distributed Bragg Reflector (DBR) below. The array is separated from the PC by a $15 \mathrm{~nm}$ thick dielectric layer of $\mathrm{Al}_{2} \mathrm{O}_{3}$ (Fig. 1(a)) to ensure that the electrical properties of the $\mathrm{THz}$ detector are not affected. The nanoantennas scatter light into the PC layer through the dipolar resonance in each antenna. ${ }^{8}$ The DBR was designed using just 5 pairs of alternating $\mathrm{Al}_{0.55} \mathrm{Ga}_{0.45} \mathrm{As}$ and GaAs layers with a periodicity of $115 \mathrm{~nm}$, producing a stop band centered on $810 \mathrm{~nm}$. As illustrated in Fig. 1(b), this hybrid cavity displays efficient absorption at the center of the stopband: $79 \%, 82 \%$, and $83 \%$ of the laser light are absorbed within the hybrid cavity for a GaAs PC layer of thickness 50, 160 , and $270 \mathrm{~nm}$, respectively. By increasing or decreasing the DBR thickness, the stop band and optimum absorption wavelength can be shifted to suit the desired application. In order to determine the most efficient design, we numerically and experimentally investigate critical parameters, varying optical cavity thickness, $L_{\text {GaAs }}$; the antenna size, $L_{a}$ and $L_{b}$; and periods within the antenna array, $a$ and $b$ (Fig. 1(c)). Due to AlGaAs growth constraints, ${ }^{23}$ the DBR contains GaAs $(55 \mathrm{~nm})$ for the high-index layers. The use of pure GaAs produces a greater amount of unwanted optical absorption within the DBR; in high performance practical applications, the GaAs layer can be replaced with low $\mathrm{Al}$ content $\mathrm{AlGaAs}$ to effectively turn off this absorption. ${ }^{8,22}$ To compare experimental observations and numerical calculations, computational models presented here assume a DBR with GaAs layers.

Effects of the cavity design on optical absorption are investigated by experimentally and numerically obtaining 
(a)

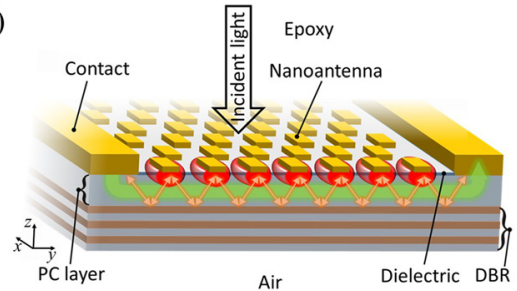

(b)

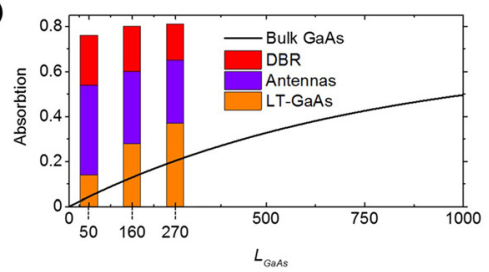

(c)

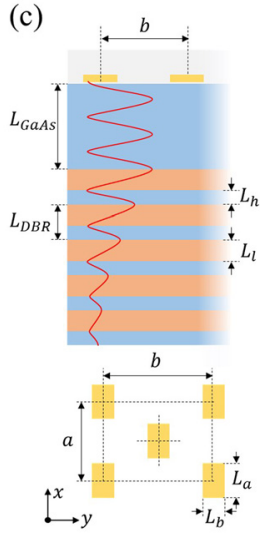

FIG. 1. (a) Optically thin photoconductive (PC) THz detector design comprising a hybrid optical cavity, bonded to a transparent substrate above by a thick layer of transparent epoxy. (b) Absorption vs. thickness for bulk GaAs (black line) and three hybrid cavities of different PC layer thickness $L_{\text {GaAs }}$. The absorption from the components of the hybrid cavity is also shown. (c) Schematic of the hybrid cavity; the red line depicts the estimated transverse electric field (absolute value) within the resonant cavity.

reflection and transmission spectra of the cavity. For numerical analysis, a commercial-grade simulator based on the finite-difference time-domain (FDTD) is used. ${ }^{24}$ All numerical analysis is undertaken by invoking the following parameters, unless otherwise stated, $L_{a}=90 \mathrm{~nm}, L_{b}=60 \mathrm{~nm}$, antenna height $=65 \mathrm{~nm}, a=250 \mathrm{~nm}$, and $b=340 \mathrm{~nm}$. Within the numerical analysis, periodic boundary conditions ensured that all scattered light was taken into consideration. Several cavity designs were fabricated by electron beam lithography. Devices were attached to a transparent substrate with an optical epoxy, and the GaAs substrate was removed. Further fabrication details can be found in the supplementary material, Section S1 and Ref. 8. The overall absorption was estimated from the reflectivity $(R)$ and transmission $(T)$ as $1-(R+T)$, to allow for comparison between modelled data and experimental data; this estimation is used throughout this letter. The proportion of absorption within each constituent component of the hybrid cavity has been modelled and is presented in the supplementary material, Section S2. Reflectivity spectra were acquired using white light illumination focused onto the sample using an objective lens $(\mathrm{NA}=0.7)$ for two polarizations defined by the array axes, $a$ and $b$. All reflectivity spectra were normalized against reference spectra acquired from a gold surface. Transmission spectra were acquired in a similar manner with another microscope objective $(\mathrm{NA}=0.5)$ positioned below the sample. The microscope NA used for characterization are similar to that used in device operation and therefore provides an accurate comparison to device performance. $\mathrm{THz}$ characterization has been undertaken by comparing a hybrid structure based detector to a detector without a nanoantenna array (see supplementary material, Section S1).

Fig. 2 shows modeled (a) and experimental (b) reflectivity and transmission spectra of the hybrid cavity. For comparison, each plot also shows spectra of the cavity without the nanoantenna array (i.e., just the DBR), where the stopband is highlighted. The hybrid cavity shows a clear suppression of reflectivity to $\mathrm{R}=\sim 5 \%$ within the DBR stop band,

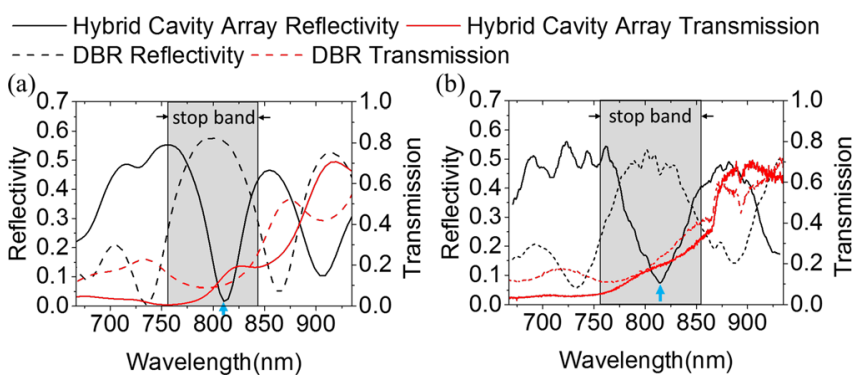

FIG. 2. Modeled (a) and experimental (b) spectra showing reflectivity (black) and transmission (red) for the hybrid cavity (solid lines) compared to the same structure with no nanoantenna array (dashed). The DBR stop band is highlighted, and the cavity mode is indicated by a blue arrow. Hybrid cavity parameters: $a=250 \mathrm{~nm}, b=350 \mathrm{~nm}, L_{\text {GaAs }} 270 \mathrm{~nm}$, electron beam current density for antenna fabrication: $500 \mu \mathrm{C} / \mathrm{cm}^{2}$.

similar to a microcavity mode; however, this is accompanied in the former case with little variation in transmission at this wavelength. These results clearly demonstrate the effect of the nanoantenna array, dramatically increasing the absorption within the cavity at $\sim 810 \mathrm{~nm}$.

In optimizing the parameters of the cavity, we first consider the PC layer thickness, $L_{\text {GaAs. }}$. Fig. 3 shows color plots of modeled reflectivity (a) and absorption (b) spectra plotted as a function of $L_{G a A s}$. Three clear areas of high absorption can be identified, each approximately confined to the DBR stop band. The absorption maxima lie at: $810 \pm 2 \mathrm{~nm}$ for a hybrid cavity with the PC layer thickness of $270 \mathrm{~nm}$; and $806 \pm 2 \mathrm{~nm}$ for thicknesses of 160 and $50 \mathrm{~nm}$. The maximum absorption within the cavity is $83 \%, 82 \%$ and $79 \%$, respectively. We note that the nanoantenna array effectively serves as an antireflection (or impedance matching) layer.
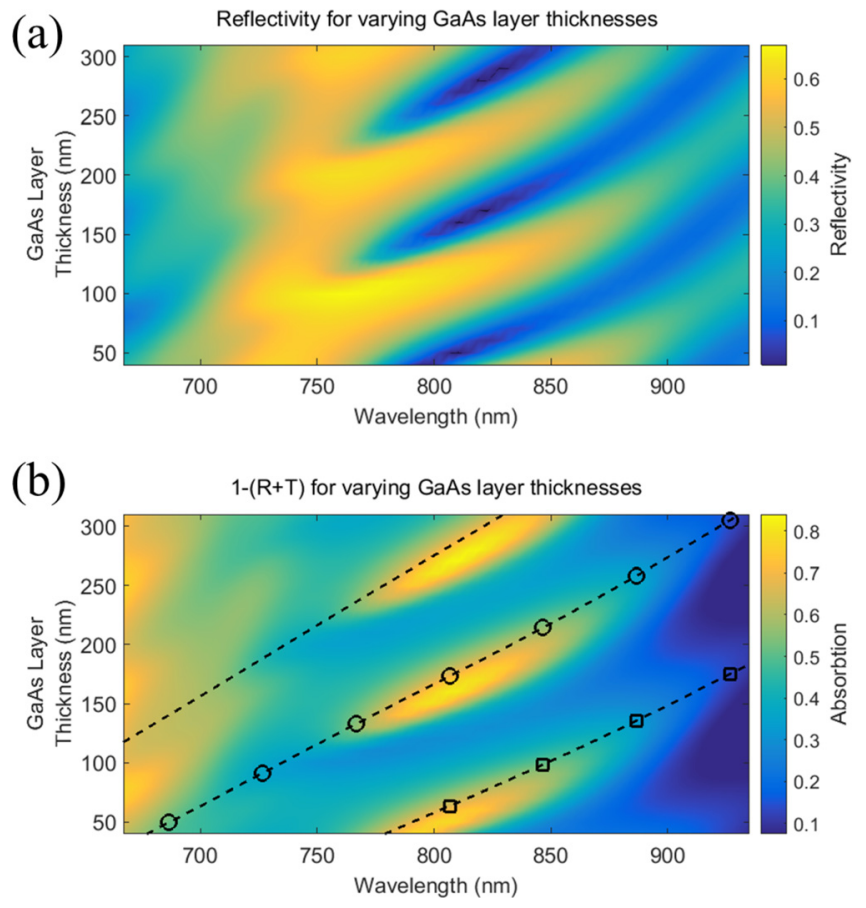

FIG. 3. Normalized reflectivity (a) and normalized absorption (b) plotted against varying GaAs layer thickness. Dashed lines in (b) plot the GaAs layer thickness, which at any given wavelength satisfies the $k$-vector conditions expressed by Eq. (1); the dashed, dashed-circle, and dashed-square lines show the $k$-vector conditions for the mode order of $m_{r}=5.5,6.5$, and 7.5 , respectively. 
To describe the effect that the PC layer thickness, $L_{\text {GaAs }}$, has on the intensity and spectral position of the absorption, we produce an intuitive picture of the electromagnetic field distribution within the device, in the $y z$ plane. Here, varying $L_{G a A s}$ changes the length of a resonant cavity and therefore the resonant wavelength. We must however take into account the entire resonant cavity consisting of the PC layer and the underlying DBR. The consequential resonance invoked within the cavity is depicted in Fig. 1(c) and can be approximated by the $k$-vector condition:

$$
k=\frac{2 \pi}{\lambda}=\frac{m_{r} \pi}{L_{G a A s} n_{G a A s}+m_{D B R} L_{D B R} n_{D B R}} .
$$

Here, $m_{r}$ is a half integer multiple representing the mode order of resonance in the cavity, $L_{\text {GaAs }}$ is the thickness of the LT-GaAs layer, $n_{\text {GaAs }}$ is the refractive index of GaAs, $m_{D B R}$ is the number of DBR pairs of thickness $L_{D B R}$, and $n_{D B R}$ is the averaged refractive index of the DBR. This $k$-vector condition is plotted in Fig. 3(b) for $m_{r}=5.5,6.5$, and 7.5. For the wavelength range $750-875 \mathrm{~nm}$, the $k$-vector condition coincides with the region of maximum absorption, confirming excitation of the cavity modes. The optimum thickness of the PC layer, therefore, can be expressed in terms of the excitation wavelength $L_{\text {GaAs }}=(1 / 4+1 / 2 N) \lambda / n_{\text {GaAs }}$, where $N=0,1,2, \ldots$ The DBR stopband (Fig. 2) coincides with the same range, therefore effectively defining wavelengths for which these cavity modes can be supported.

It was suggested in Ref. 8 that periodicity of the nanoantenna array can affect the coupling of light into the cavity modes. To investigate this, Fig. 4 displays numerically calculated and experimentally acquired spectra for hybrid cavities with differing nanoantenna array periodicity. Fig. 4(a) shows SEM images of two arrays with periods $a=350 \mathrm{~nm}$ and $450 \mathrm{~nm}$. Surprisingly, the cavity mode exhibits practically no shift in the wavelength and only a small change in absorption within a wide range of periods. For the array periods larger than the effective wavelength $\left(\lambda / n_{\mathrm{GaAs}}\right)$, additional modes appear in the absorption maps (Figs. 4(b) and 4(c)) with characteristic diagonal traces across the map. Similar effects were seen by Munday et al. with antireflective grating structures on silicon for photovoltaic applications. ${ }^{25}$ For further investigation, the calculated wavelength of the absorption peak and level of absorption are plotted as maps in Figs. 4(d) and $4(\mathrm{e})$. The cavity mode wavelength remains practically constant, and the high value of absorption is maintained within a large parameter space. At the bottom left corner of Figs. 4(d) and 4(e), where $k_{y}^{2}+k_{x}^{2}<k_{0}{ }^{2} n^{2}$, the peak wavelength changes and the absorption is reduced, indicating the presence of other modes. Detailed investigation of all modes in the hybrid cavity is beyond the scope of this paper. Here, however, we emphasize that efficient absorption in the hybrid cavity can be achieved for a range of arrays with periods $a$ and $b$ that satisfy approximately the following mathematical expression: $(2 \pi / a)^{2}+(2 \pi / b)^{2}=k_{0}{ }^{2} n^{2}$. Fig. 4(f) illustrates experimental verification of the robustness of the mode wavelength for arrays with the period variation of $100 \mathrm{~nm}$.

Despite the broad linewidth $(\sim 200 \mathrm{~nm})$ of the plasmonic resonance of the individual antennas, ${ }^{8}$ we find the antenna length to be a sensitive parameter of the hybrid cavity. Fig. 5(a) shows modeled reflectivity spectra for a range of antenna lengths $L_{a}$. The $y$ and $z$ dimensions of the antenna were kept constant. A red-shift of the reflectivity dip was observed for larger $L_{a}$ with minimum reflectivity (maximum absorption) occurring at $L_{a} \approx 90 \mathrm{~nm}$. To verify this effect experimentally, the antenna size was varied by altering the electron beam dosage used during lithography. Fig. 5(b) shows SEM images of two arrays produced at beam dosages of $450 \mu \mathrm{C} / \mathrm{cm}^{2}$ and $600 \mu \mathrm{C} / \mathrm{cm}^{2}$. Fig. 5(c) shows the reflectivity spectra for these dosages and also for a dosage of $550 \mu \mathrm{C} /$ $\mathrm{cm}^{2}$ with the red shift at larger antenna sizes agreeing with the modeled data.

The strong antenna size effect (Fig. 5) suggests that the array not only scatters incident photons into the cavity but also enables matching of the input impedance to the freespace impedance minimizing reflection. The total reflection from the hybrid cavity can be intuitively approximated as a superposition of two components: the wave reflected by the DBR and the wave reflected by the antennas. Within the DBR stopband, the phase of the former is approximately constant, whereas the phase of the latter varies with wavelength rapidly due to the antenna resonance. Therefore, the two components can be out of phase and interfere destructively, leading to suppression of reflection. This explains why the antenna size and the GaAs layer thickness affect the optimum wavelength. We note that the concept of impedance matching is used in metamaterial perfect absorbers, where the surface impedance can be tuned by tailoring electrical and magnetic resonances. ${ }^{26,27}$ It has also been shown that perfect absorption can be achieved in layered metamaterials without effective magnetic resonance via multiple reflections. ${ }^{28}$ Our results indicate a similar mechanism of impedance matching for the hybrid cavity considered here.

$\mathrm{THz}$ detectors with the hybrid cavity design described above were fabricated and compared to identical detectors without the nanoantenna array. The relative $\mathrm{THz}$ electric field amplitude for the detectors with and without the nanoantenna array shows the enhancement of the $\mathrm{THz}$ response near the wavelength of the hybrid cavity resonance, (see supplementary material, Section S1 for experimental details). Figure 6(a) shows the waveform of a THz pulse generated using a ZnTe crystal and silicon lens (inset). The waveform was detected using $\mathrm{THz}$ detectors with the hybrid cavity (red trace) and also a cavity without the nanoantenna array (black trace). In each case, the detector was optically gated by a Ti:sapphire laser tuned to $815 \mathrm{~nm}$. The pulse waveform in both cases is the same; however, the amplitude of the detected field was lower for the case of the detectors without the nanoantenna array. To investigate the variation of the detector performance with the wavelength of the gating laser pulses, THz detection experiments were repeated for various laser wavelengths. To avoid $\mathrm{THz}$ beam misalignment due to the optical wavelength tuning, a $1 \mu \mathrm{m}$ thick layer of InAs grown on GaAs was used for generation of $\mathrm{THz}$ pulses (inset). This configuration produces an unfocused $\mathrm{THz}$ beam with a larger cross-section compared to the configuration with the Si lens, while at the same time, the GaAs layer blocks the laser beam from reaching the detectors. The 
(a)

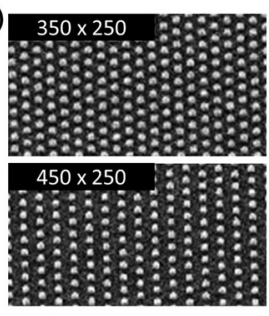

(b)

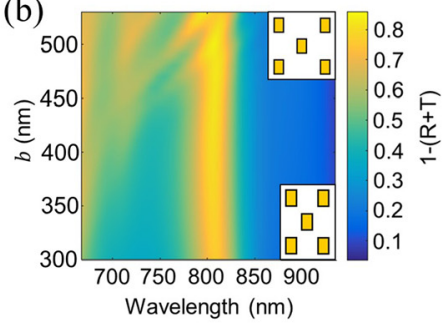

(e)

(d)

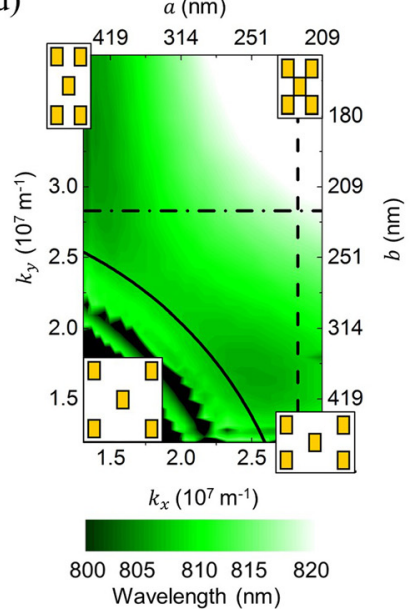

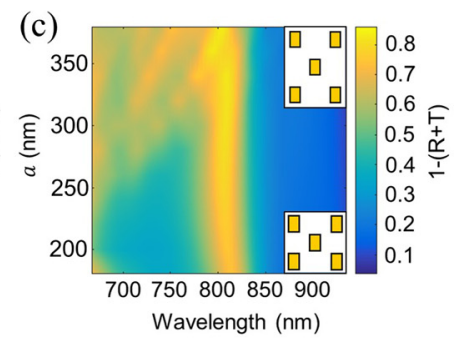

(f)

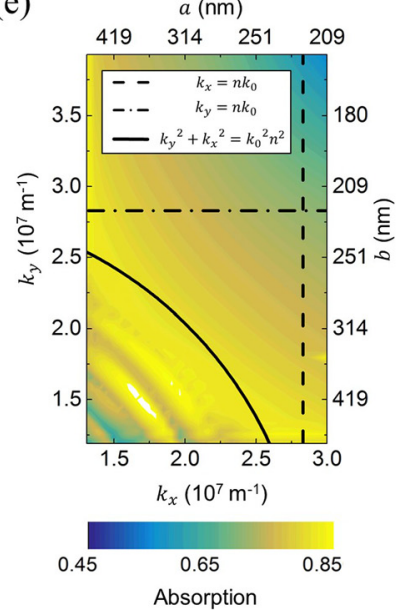

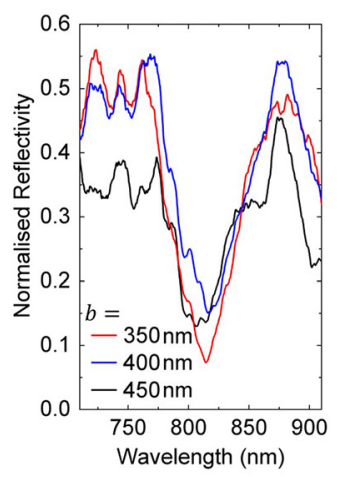

FIG. 4. Variation in absorption due to nanoantenna periodicity. (a) Nanoantenna array SEM images of hybrid cavities with parameters: $L_{\text {GaAs }}$ $=270 \mathrm{~nm}$, electron beam current density: $500 \mu \mathrm{C} / \mathrm{cm}^{2}$. (b), (c) Absorption spectra as functions of varying period in $y$ and $x$, respectively. (d) Absorption peak wavelength and (e) normalized peak absorption as functions of periodicity in both $x$ and $y . L_{\text {GaAs }}=50 \mathrm{~nm}$. (f) Experimental reflectivity spectra for cavities displayed in (a). relative $\mathrm{THz}$ electric field amplitude for the detectors with and without the nanoantenna array is plotted in Fig. 6(b). The detectors with the nanoantenna array show enhancement of the $\mathrm{THz}$ response near the wavelength of the hybrid cavity resonance. This enhancement in sensitivity $(\sim 17 \%)$ is however lower than a relative increase in absorption in the LT GaAs layer (from $23 \%$ to $38 \%$ ) predicted by our numerical simulations (see Table S1, the supplementary material). The spectral bandwidth characteristics for both detectors are similar and shown in supplementary material.

Despite the optimization of overall absorption presented within this paper, absorption in the active layer alone must be optimized further to develop more efficient detectors. Our simulations indicated significant optical absorption within both the nanoantennas and the DBR [Fig. 1(b)]. As mentioned previously, absorption within the DBR can be (a)

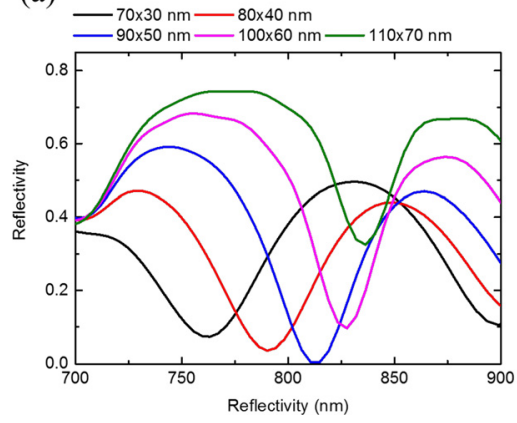

(b)

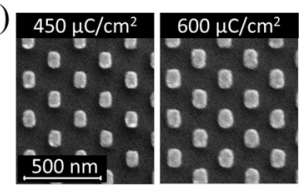

(c)

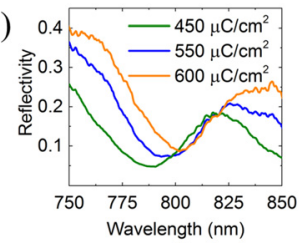

FIG. 5. (a) Modelled reflectivity spectra at varying antenna sizes, $L_{a}$ and $L_{\mathrm{b}}$ listed in the legend, $L_{G a A s}=50 \mathrm{~nm}$. (b) SEM images of nanoantenna, samples with electron beam exposure during manufacture, here $L_{\text {GaAs }}=270 \mathrm{~nm}$, $a=250 \mathrm{~nm}$ and $b=350 \mathrm{~nm} .450 \mu \mathrm{C} / \mathrm{cm}^{2}$ and $600 \mu \mathrm{C} / \mathrm{cm}^{2}$ e-beam dosages produced antenna dimensions of $L_{a}=100 \mathrm{~nm}, L_{\mathrm{b}}=70 \mathrm{~nm}$ and $L_{a}=110 \mathrm{~nm}$, $L_{\mathrm{b}}=80 \mathrm{~nm}$, respectively. (c) Reflectivity spectra of the cavities displayed in (b). eliminated almost entirely if the GaAs is replaced with a low $\mathrm{Al}$ content $\mathrm{AlGaAs}$ layers, e.g., $\mathrm{Al}_{0.2} \mathrm{Ga}_{0.8} \mathrm{As} .{ }^{22}$ For a $270 \mathrm{~nm}$ hybrid cavity, optical absorption within the PC layer increases from $38 \%$ to $48 \%$ for the case of a non-absorbing DBR, see supplementary material. Unwanted absorption within the nanoantenna array may be decreased by (1) using metals with lower absorption, and (2) by reducing the density of nanoantennas. Figure 4 shows that when the density is decreased to produce a period above $a=300 \mathrm{~nm}$ and $b=400 \mathrm{~nm}$ [Figs. 4(b) and 4(c)], optical absorption remains high, implying increased absorption within the PC layer. However, additional modes are seen in the absorption spectra [Fig. 4]; the effects of these modes have been avoided thus far, and further study is required to understand their potential beneficial effects. At higher periodicities, interference of these with the hybrid cavity mode could be manipulated to harness higher absorption within the PC layer.

In conclusion, we provide an analysis of the key parameters for the design of optically thin, highly absorbing, photoconductive (PC) structures for $\mathrm{THz}$ detectors. We find that a device operating at $810 \mathrm{~nm}$, with a PC layer of $50 \mathrm{~nm}$ (one quarter the operating wavelength in GaAs), a 5 period DBR stack, and a nanoantenna array produced a hybrid cavity with peak optical absorption of 79\%. In designing the cavity, we find that the developed analytical model predicts the absorption wavelength dependence on the PC layer thickness with the optimum values being expressed in terms of the excitation wavelength. These optimum PC layer thicknesses coincide with odd quarter multiples $(1 / 4,3 / 4,5 / 4)$ of the operating wavelength in GaAs; however, with the larger multiples, a marginal increase in absorption within the hybrid cavity occurs. We further find that combined squared periodicities of the nanoantenna array should approximately equal the squared wave vector of the operating wavelength, 

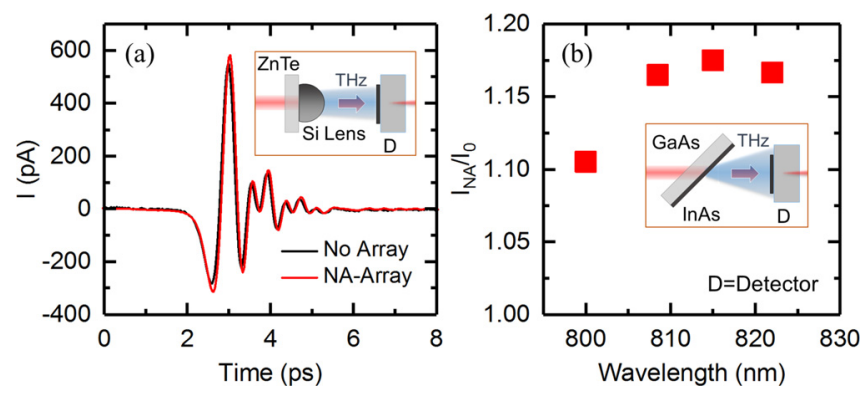

FIG. 6. THz pulse detection using PC detectors (D) with and without the nanoantenna (NA) array. (a) Waveforms of the THz pulse measured by the hybrid cavity $\mathrm{THz}$ detector (red) and a similar detector without the antenna array (black). (b) The electric field amplitude detected by the hybrid cavity $\mathrm{THz}$ detector relative to the detector without the nano-antenna array as a function of the central laser wavelength. Insets in (a) and (b) show the experimental arrangements. (Full experimental details can be found in the supplementary material.)

and care should be taken in choosing the nanoantenna size, which affects both the operating wavelength and efficiency. We also note that absorption within the nanoantennas is nonnegligible, and further designs of hybrid cavities should aim to address this.

See supplementary material for a detailed description of the fabrication method used (Section S1) along with terahertz characterization of the hybrid cavity detector and for a comparison of a detector without a nanoantenna array. Within section S2, the power absorption of constituent components in the cavity, as presented in Fig. 1(b), is tabulated, and for comparison, a hybrid cavity with a nonabsorbing DBR and a cavity with no nanoantennas are also presented.

This work was supported by the Royal Society [Grant No.: UF130493] and EPSRC [Grant No.: EP/M507970/1]. The work was performed at UCL and in part at the Center for Integrated Nanotechnologies, an Office of Science User Facility operated for the U.S. Department of Energy (DOE) Office of Science. Sandia National Laboratories is a multiprogram laboratory managed and operated by Sandia Corporation, a wholly owned subsidiary of Lockheed Martin Corporation, for the U.S. Department of Energy's National
Nuclear Security Administration under Contract No. DEAC04-94AL85000.

${ }^{1}$ Y. Zhang, K. Shibata, N. Nagai, C. Ndebeka-Bandou, G. Bastard, and K. Hirakawa, Appl. Phys. Lett. 107, 103103 (2015).

${ }^{2}$ K. Peng, P. Parkinson, L. Fu, Q. Gao, N. Jiang, Y. Guo, F. Wang, H. J. Joyce, J. L. Boland, H. H. Tan, C. Jagadish, and M. B. Johnston, Nano Lett. 15, 206 (2015).

${ }^{3}$ M. S. Vitiello, D. Coquillat, L. Viti, D. Ercolani, F. Teppe, A. Pitanti, F. Beltram, L. Sorba, W. Knap, and A. Tredicucci, Nano Lett. 12, 96 (2012).

${ }^{4}$ M. Mittendorff, S. Winnerl, J. Kamann, J. Eroms, D. Weiss, H. Schneider, and M. Helm, Appl. Phys. Lett. 103, 021113 (2013).

${ }^{5}$ X. Cai, A. B. Sushkov, M. M. Jadidi, L. O. Nyakiti, R. L. Myers-Ward, D. K. Gaskill, T. E. Murphy, M. S. Fuhrer, and H. D. Drew, Nano Lett. 15, 4295 (2015).

${ }^{6}$ L. Viti, D. Coquillat, A. Politano, K. A. Kokh, Z. S. Aliev, M. B. Babanly, O. E. Tereshchenko, W. Knap, E. V. Chulkov, and M. S. Vitiello, Nano Lett. 16, 80 (2016).

${ }^{7}$ M. Jarrahi, IEEE Trans. Terahertz Sci. Technol. 5, 391 (2015).

${ }^{8}$ O. Mitrofanov, I. Brener, T. S. Luk, and J. L. Reno, ACS Photonics 2, 1763 (2015)

${ }^{9}$ C. W. Berry, N. Wang, M. R. Hashemi, M. Unlu, and M. Jarrahi, Nat. Commun. 4, 1622 (2013).

${ }^{10}$ J. Požela and A. Reklaitis, Solid-State. Electron. 23, 927 (1980).

${ }^{11}$ S. Kono, M. Tani, P. Gu, and K. Sakai, Appl. Phys. Lett. 77, 4104 (2000).

${ }^{12}$ C. W. Berry, M. R. Hashemi, and M. Jarrahi, Appl. Phys. Lett. 104, 081122 (2014).

${ }^{13}$ B. Heshmat, H. Pahlevaninezhad, Y. Pang, M. Masnadi-Shirazi, R. B. Lewis, T. Tiedje, R. Gordon, and T. E. Darcie, Nano Lett. 12, 6255 (2012).

${ }^{14}$ S. Matsuura, M. Tani, and K. Sakai, Appl. Phys. Lett. 70, 559 (1997).

${ }^{15}$ K. Moon, I.-M. Lee, J.-H. Shin, E. S. Lee, N. Kim, W.-H. Lee, H. Ko, S.-P. Han, and K. H. Park, Sci. Rep. 5, 13817 (2015).

${ }^{16}$ Q. Y. S. Wu, H. Tanoto, L. Ding, C. Choy Chum, B. Wang, A. B. Chew, A. Banas, K. Banas, S. Jin Chua, and J. Teng, Nanotechnology 26, 255201 (2015).

${ }^{17}$ J. L. Briscoe and S.-Y. Cho, Electron. Lett. 50, 1860 (2014).

${ }^{18}$ H. A. Atwater and A. Polman, Nat. Mater. 9, 205 (2010).

${ }^{19}$ S. J. Kim, I. Thomann, J. Park, J. H. Kang, A. P. Vasudev, and M. L. Brongersma, Nano Lett. 14, 1446 (2014).

${ }^{20}$ C. Simovski, D. Morits, P. Voroshilov, M. Guzhva, P. Belov, and Y. Kivshar, Opt. Express 21, A714 (2013).

${ }^{21}$ S. Collin, F. Pardo, and J.-L. Pelouard, Appl. Phys. Lett. 83, 1521 (2003).

${ }^{22}$ A. J. Macfaden, J. L. Reno, I. Brener, and O. Mitrofanov, Appl. Phys. Lett. 104, 011110 (2014).

${ }^{23}$ The MBE growth was performed with a single Al cell.

${ }^{24}$ See http://www.lumerical.com/tcad-products/fdtd/ for Lumerical Solutions, Inc.

${ }^{25}$ J. N. Munday and H. A. Atwater, Nano Lett. 11, 2195 (2011).

${ }^{26}$ Y. Ra'di, C. R. Simovski, and S. A. Tretyakov, Phys. Rev. Appl. 3, 037001 (2015).

${ }^{27}$ J. Hao, J. Wang, X. Liu, W. J. Padilla, L. Zhou, and M. Qiu, Appl. Phys. Lett. 96, 251104 (2010).

${ }^{28}$ H.-T. Chen, Opt. Express 20, 7165 (2012). 